\title{
Assessment of Percolation Threshold Simulation for Individual and Hybrid Nanocomposites of Carbon Nanotubes and Carbon Black
}

\author{
Paulo Henrique da Silva Leite Coelho ${ }^{a}$, Victor Antonio de Deus Armellini ${ }^{b}$, Ana Rita Morales ${ }^{b}$ \\ ${ }^{a}$ Chemical Engineering, Federal Univesity of Maranhao, 65080-805, São Luis, MA, Brazil. \\ ${ }^{b}$ School of Chemical Engineering, State University of Campinas, 13083-852, Campinas, SP, Brazil.
}

Received: December 14, 2016; Revised: June 28, 2017; Accepted: August 17, 2017

\begin{abstract}
Modeling electrical conductivity of polymer composites with conductive fillers has great applicability to predict conductive materials behavior. In this study, the electrical behavior of simple and hybrid systems prepared from Carbon Black (CB) and Carbon Nanotubes (CNT) was studied. There have been few advances reported in the literature regarding the modeling of hybrid systems, which motivated the development of this study. More specifically, a program was developed with the intention to describe the electric percolation threshold and the effect of synergism between the conductive fillers. Simulation was performed using the Monte Carlo method and Fortran programming language, considering concentration and geometry of conductive fillers to the system in two dimensions. Finally, simulation results were compared with the experimental results and this method proved to be effective in predicting the systems percolation threshold, being an important contribution to predict material behavior, which allows reducing the number of samples to be prepared in an experimental study.
\end{abstract}

Keywords: Percolation, Polymer nanocomposites, Simulation, Hybrid fillers, Monte Carlo method.

\section{Introduction}

Adding electrically conductive particles such as carbon black (CB) and carbon nanotubes (CNT) above critical concentrations in an insulating polymer resin has been a resource for obtaining conductive composites ${ }^{1}$.

Combining different carbon fillers is an alternative for obtaining a system with balanced properties and costs. The mixture of these types of fillers can be more efficient when forming conductive networks, since fillers with high aspect ratios such as CNT can act as carriers for electrical fillers, and particles with lower aspect ratio such as $\mathrm{CB}$ can have the function of filling the spaces, promoting interconnection between the nanotubes in the polymer compound. The different characteristics of carbon conductive fillers may provide, to the composite, an electrical percolation threshold at lower concentrations, when compared to composites with only one type of filler ${ }^{2,3}$.

The main issue about the conductive polymer nanocomposites is the conductivity variation as function of conductive particles concentration, where a critical concentration can be observed, from which an increase in the system conductivity occurs. This phenomenon can be explained by percolation theory. Several percolation models have been developed to describe this phenomenon, and there is a comprehensive review described in the literature ${ }^{4}$. However, almost all of these models describe the phenomena to only one type of

*e-mail: piyke.coelho@gmail.com conductive filler, and very little has been discussed on a suitable model for systems with different conductive carbon particles. The works of Sun et al., ${ }^{5}$ and Socher et al., ${ }^{6}$ described, from the excluded volume theory, electrical percolation of individual CNT and CB fillers and their mixtures as a function of volume fraction of these particles.

Among the methods used in the simulation of these models that describe percolation, the Monte Carlo method has provided satisfactory results since it is associated with the generation of random numeric data for obtaining values deterministically difficult to define ${ }^{7}$. It has been widely used in solving several models in which an analytical resolution cannot be found.

The evaluation of percolation threshold of simulated systems consists in randomly generate a predefined fraction of elements defined as conducting particles, in a fully insulating matrix of suitable size. This procedure is derived from the classical percolation theory by site $^{8}$, and leads to a theoretical value for the percolation threshold, which is generally defined by square lattices (two-dimensional systems) or cubic (three-dimensional systems) ${ }^{9}$. The matrix fraction occupied by conductive elements is provided to the program, representing the fraction of the volume of conductor additive in the composite. Upon reaching a concentration where an interconnected network of conducting elements is formed, the program encounters the critical value called the percolation limit $\left(p_{c}\right)^{4}$.

The vast majority of theoretical works cited in the literature ${ }^{10-13}$ describes models in which conductive fillers 
are considered to be straight or rigid rods. However, this is not the CNT structure reality. The flexibility in these existing fillers and high aspect ratio form interlaced nanotubes in the polymer matrix. This structure complicates the modeling and simulation process because the fitting leads to a diversity of particles configuration.

In order to create a simulator, some important aspects must be defined, such as the core feature, which determines how the simulator will handle the overlapping particles. While many modeling studies consider CNT as permeable cylinders or with "soft core", assuming that the electrical percolation threshold and the geometric percolation occur simultaneously ${ }^{9}$, other studies consider a more realistic situation: CNT has no direct overlap with each other, being called "hard core", and are wavy instead of rigid rods ${ }^{14,15}$. In simulation by Monte Carlo method it is possible to observe an inverse proportionality between the percolation threshold and the excluded volume for both "soft core" and "hard core". But, the "hard core" model is more appropriate for modeling composites where the mechanism of electrical conduction is by tunneling. Furthermore, the proportionality constant was found to be governed by the fillers aspect ratio ${ }^{14}$. Considering a system where fillers are fiber-shaped, if these are highly curved or even curled, there is a significant difference in the modeling for rigid or flexible fibers. For shorter fibers, the curvature effect is much more pronounced than for longer fibers in percolation threshold prediction ${ }^{15}$.

The "hard-core" model is considered to be more faithful to the laws of physics; however, the use of "soft-core" model is more common in the literature since it is simpler to be implemented and requires less computing resources. However not always the results obtained by this model are close to the experimental results and their use should be evaluated in each case ${ }^{15}$.

Another important parameter to consider is spatial discretization, which is a consequence for the use of a discrete matrix to represent the full or empty sites.

Due to discretization a fiber or tube can be represented as a zig-zag parallel or orthogonal segment to the matrix axes (Figure 1), which represents the discretized space and is similar to the curling behavior of the fibers as previously discussed in the literature ${ }^{15,16}$. These works consider that this curling can affect the percolation threshold. The discussion is supported by theoretical analysis of curling fibers influence in percolation threshold, showing that the curling effect in the percolation threshold is negligible at aspect ratios smaller than 100, and even for larger aspect ratios the effect is small.

For $\mathrm{CB}$, particles in the insulating matrix does not generate a concern regarding the discretization. Typically, these particles are considered as spherical; however, what apparently seems to facilitate the simulation, in fact the filler geometry leads to a difficulty, since $\mathrm{CB}$ particles are always aggregated presenting different conformations in the matrix.

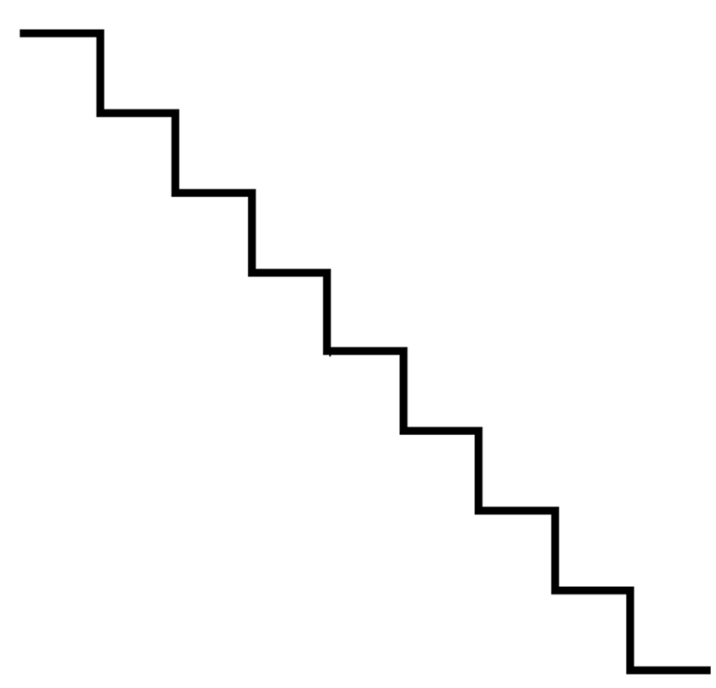

Figure 1. Scheme of CNT discretized.

Considering the potential application for filler hybrid systems and that investigations are still necessary concerning the synergism effects between fillers, the aim of this study was to develop a program that simulates percolation threshold for particles with different aspect ratios. The study was based on Monte Carlo method, supported by excluded volume model, and programmed in Fortran language. The program was developed in order to fit the percolation threshold of nanocomposites experimentally prepared with individual $\mathrm{CB}$ and CNT fillers and with fillers blends, called hybrid systems. The results of the mathematical simulation were compared with those obtained for a polymer matrix of PMMA. This work considered a perfect system, which excluded the effect of parameters that could influence the behavior of conductive composites. We considered the particles to be not agglomerated, i.e. individual, an average aspect ratio, a perfect dispersion and a homogeneous system.

\section{Materials and Methods}

The CNT material was a multi wall carbon nanotube, TNM2 from Chengdu Organic Chemicals Co. Ltd fabricated by chemical vapor deposition, with $95 \%$ of purity, average external diameter of $11.5 \mathrm{~nm}$ and average length of $50 \mu \mathrm{m}$. The conductive Carbon Black (CB) was high structure Printex $\mathrm{XE2}$, from Evonik Degussa $\mathrm{GmbH}$, average particle size of $30 \mathrm{~nm}$. The monomer was the methyl methacrylate (MMA) M/4950/08 from Fischer Scientific that was previously purified to remove hydroquinone. The solvent employed was acetone PA-ACS from QUEMIS. The polymerization was conducted in solution of acetone PA-ACS from QUEMIS in presence of the initiator 2,2'-Azobys (2-methylpropionitrile) (AIBN) Vaso 64, DuPont.

Pre-mixtures were prepared by adding different concentrations of fillers into glass tubes containing acetone, 
MMA, and AIBN. The solutions were purged with $\mathrm{N}_{2}$, and then submitted to ultrasonic stirring using a UP400S probe (Hielscher) at $100 \mathrm{~W}$ and constant pulse frequency of $24 \mathrm{KHz}$ for 20 minutes. To complete the polymerization, the samples were kept at $80{ }^{\circ} \mathrm{C}$ for 24 hours. The films are obtained by casting with diameter of $55 \mathrm{~mm}$ and thickness of $0.5 \mathrm{~mm}$, approximately. In order to calculate the fillers volume fraction (V) the density of the fillers were determined by gaseous pycnometry, and for PMMA, by liquid pycnometry. Singleconductive-filler filled systems (SCFFS) formulations were previously studied to obtain the $p_{\mathrm{c}}$ published as $p_{\mathrm{c}}=0.2 \mathrm{vol} . \%$ for $\mathrm{CNT}^{17}$ and $p_{\mathrm{c}}=1.5 \mathrm{vol} . \%$ for $\mathrm{CB}^{18}$. From these $p_{\mathrm{c}}$ and $\mathrm{CB}$ fillers were prepared as shown in Table 1. The Sun's model, described by Equation 1 was used to describe the condition for additive behavior, i.e., the MCFFS presents percolation ${ }^{5}$.

$$
\frac{V_{C N T}}{p_{c, C N T}}+\frac{V_{C B}}{p_{c, C B}}=1
$$

where $p_{c^{\prime} C N T}$ and $p_{c^{\prime} C B}$ are percolation threshold of single fillers systems, and $V_{\mathrm{CNT}}$ and $V_{\mathrm{CB}}$ are individual volumetric fraction of respective fillers.

Table 1. Volumetric fraction (V) (\%) of CNT and CB in the MCFFS

\begin{tabular}{lccc}
\hline Samples & $\mathrm{V}_{\mathrm{CNT}}$ & $\mathrm{V}_{\mathrm{CB}}$ & $\mathrm{V}_{\mathrm{CNT}}+\mathrm{V}_{\mathrm{CB}}$ \\
\hline 1 & 0.03 & 1.27 & 1.3 \\
2 & 0.06 & 1.07 & 1.13 \\
3 & 0.11 & 0.64 & 0.75 \\
4 & 0.17 & 0.21 & 0.38 \\
5 & 0.01 & 1.21 & 1.22 \\
6 & 0.03 & 0.91 & 0.94 \\
7 & 0.06 & 0.6 & 0.66 \\
8 & 0.11 & 0.3 & 0.41 \\
\hline
\end{tabular}

MCFFS were prepared by varying $V_{\mathrm{CB}}$ for a fixed $V_{\mathrm{CNT}}$. Total filler concentration on the systems was always below the sum of the critical concentration of the individual fillers, i.e., $p_{\mathrm{c}, \mathrm{CNT}}+p_{\mathrm{c}, \mathrm{CB}}<1.7$ vol. (\%). To test the Sun's model for our system we tested different scenarios. Samples 1-4 from Table 1 represent the additive condition $V_{\mathrm{CNT}} / p_{\mathrm{c}, \mathrm{CNT}}+V_{\mathrm{CB}} / p_{\mathrm{c}, \mathrm{CB}}$ $=1$; when $V_{\mathrm{CNT}} / p_{\mathrm{c}, \mathrm{CNT}}+V_{\mathrm{CB}} / p_{\mathrm{c}, \mathrm{CB}}>1$, the conductive fillers connect to each other, and the material is conductive; when $V_{\mathrm{CNT}} / p_{\mathrm{c}, \mathrm{CNT}}+V_{\mathrm{CB}} / p_{\mathrm{c}, \mathrm{CB}}<1$, the conductive fillers separately disperse in the polymer matrix, and the electrical conductivity is not expected and samples 5-8 represent this condition.

Volumetric electrical conductivity was determined by a four-point probe test, Jandel Universal Probe RM3, supplied with a $100 \mathrm{Ohm}$ test resistor and with aligned probes and $0.1 \mathrm{~cm}$ spacing. Measurements were made in the range of $10 \mathrm{nA}-10 \mu \mathrm{A}$.

Morphology was studied by using a High Resolution Transmission Electron Microscopy (HR-TEM) JEOL 3010 (tension of $300 \mathrm{kV}$ ) for films of around $40 \mathrm{~nm}$ in thickness obtained in an Ultramicrotome LEICA model ULTRACUT - UCT 020, cutting speed of $1 \mathrm{~mm} / \mathrm{s}$ using a diamond knife Diatome, Cryo-Wet.

\section{Simulation}

The simulation algorithm for the Monte Carlo method was programmed in FORTRAN language (Frtran 95 - Silverfrost Plato Version 4.61).

The program was developed to simulate percolation threshold, $p_{c}$, of two-dimensional square matrices for particles with different shapes and aspect ratios. For comparison of simulated particles geometries with experimental particles, it was considered that each point of the matrix would fill the space referring to $10 \mathrm{~nm}$. Thus, the adoted aspect ratio of 1000 for the CNT was represented by a diameter of 10 $\mathrm{nm}$ and a length of $1 \mu \mathrm{m}$.

A two-dimensional square matrix with $n \times n$ elements was associated to insulating matrix of experimental PMMA, and simulated for different values of $n$.

The $2 \mathrm{D}$ square network of $n \times n$ elements was defined as follows:

- $\quad$ "0" represents the polymer matrix (insulating elements);

- $\quad$ "1" represents the fillers (conductive elements).

The first step was constructing an insulating matrix network formed by elements 0 . After generation, the algorithm converts a fraction initially set by the user in conductive elements randomly positioned by a generator of random numbers (Monte Carlo method) in the conductive matrix. This fraction represents the amount of conductive fillers present in the polymer. Three simulators were developed, one for each filler and another to hybrid systems, where the difference was the fillers geometry. CNT were considered to have length equal to their aspect ratio and diameter as the matrix point value. In order to equalize the dimensional representation between the fillers, the $\mathrm{CB}$ aggregates were considered square whose side value is a multiple of point value, which in turn was assumed equal to the CNT diameter.

\section{Results and Discussion}

\subsection{Experimental}

PMMA-based SCFFS and MCFFS containing CNT and $\mathrm{CB}$ were developed aiming to analyze the electrical percolation threshold $\left(p_{c}\right)$, the conductivity values and the dispersion of the fillers. Figure 2 shows the MCFFS and SCFFS electrical conductivity. The results for the single systems are published elsewhere ${ }^{17,18}$ and were shown here to compare the different systems. Samples 1-4 presented electrical conductivity while for samples 5-8 no conductivity was observed, evidencing that Sun's model fits the studied systems. 


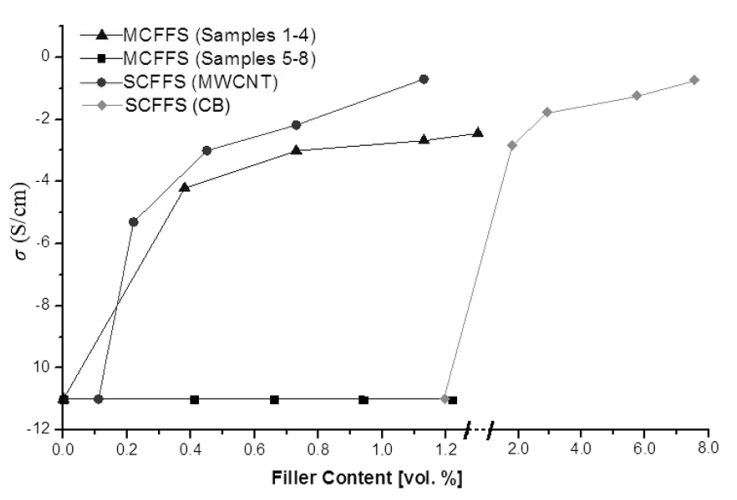

Figure 2. Experimental volumetric electrical conductivity as a function of MCFFS and SCFFS concentrations.

In order to determine the critical percolation threshold on electrical conductivity, for MCFFS the power relationship was applied (Eq. 2) on the double logarithmic graphic $\log$ $\sigma v s . \log \sigma\left(p-p_{c}\right)$.

$$
\sigma \alpha\left(p-p_{c}\right)^{t}
$$

where the percolation threshold $\left(p_{\mathrm{c}}\right)$ was obtained relating the volumetric electrical conductivity $(\sigma)$ to the volumetric concentration of conductive filler into the matrix $(p)$. The $t$ is the critical exponent and is related to the network dimensional aspects. Figure 2 shows the results.

The experimental $p_{\mathrm{c}}$ value was 0.195 vol. (\%) $\left(V_{\mathrm{CNT}}\right.$ $+V_{\mathrm{CB}}$ ). In general, the critical exponent $t$ depends on the system dimension and depends strongly on the particle type, whose value is set between 1.65 and 2 for three-dimensional network ${ }^{19-21}$. However, for CB composites, the $t$ obtained was $1.18 \pm 0.09$ for a regression coefficient of 0.96 . This difference occurred due the complex geometry and the structure of the aggregates formed and to the conduction effect between the aggregates ${ }^{1,20,22}$. For CNT, $t=2.34 \pm 0.19$, for a regression coefficient of 0.94 , which is an evidence of a three-dimensional conductive network as already found by Kim et al. ${ }^{23}$, that related critical exponent $t$ of 2.4 and $p_{\mathrm{c}}=0.3 \mathrm{wt} \%$ for CNT/PMMA composites. For MCFFS, the $t$ value is not usually found in the literature. However, as the nanotubes are mainly responsible for the percolative network formation, we can compare the $t$ with the values found in other studies. The range of critical exponent values from experimental measurements obtained by different situations indicates that $t$ is not universal. For CNT, Bauhofer and Kovacs ${ }^{1}$ have related experimental $t$ values for threedimensional percolating systems between 1.3 and 4 , which would be consistent with the MCFFS value $t=2.21 \pm 0.15$. Other studies have also shown that the percolation threshold is dependent of conductive fillers morphology (size, shape and the aspect ratio) $)^{24-27}$.

\subsection{Simulation}

\subsubsection{Verification of Simulator for scattered points.}

In order to evaluate the simulator capacity to generate random numbers, the initial tests on the simulator were performed assuming fillers as individual points of the matrix lattice, according to the routine described hereinafter for which Figure 3 illustrates the program response of a 5X5 elements matrix with the concentration of $60 \%$ fillers.

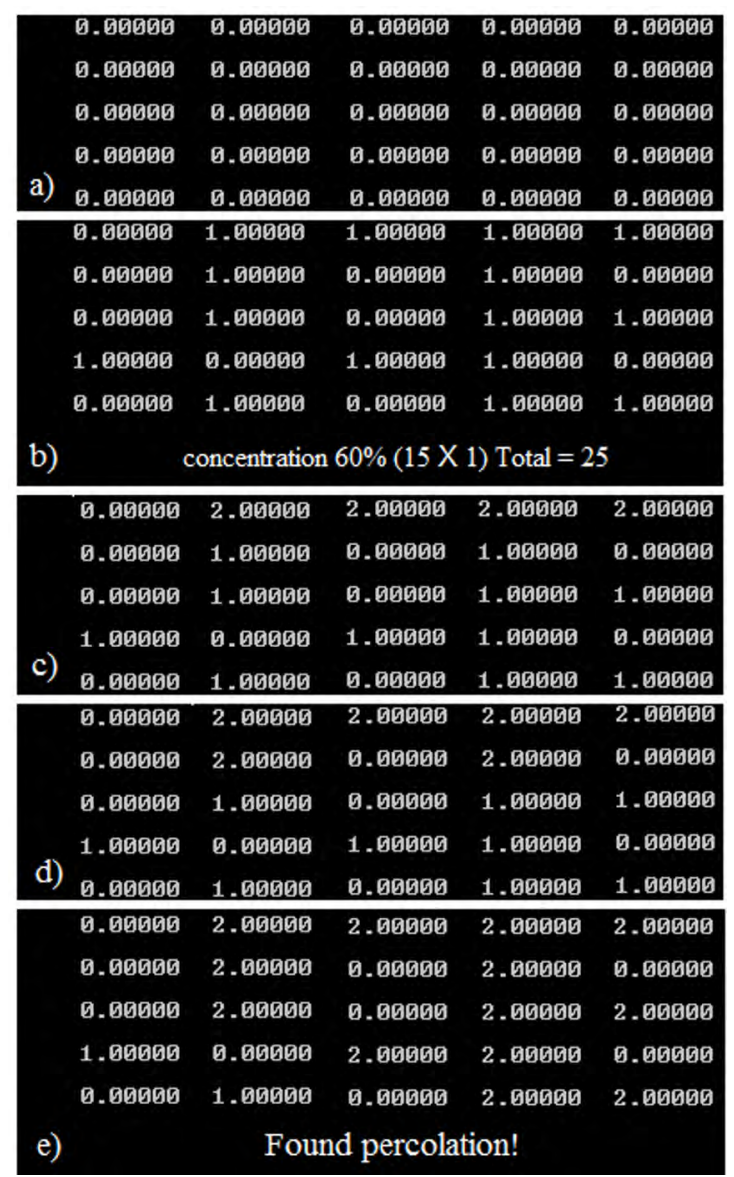

Figure 3. Matrices of verifier of Simulator of elements $5 X 5$ with the concentration of $60 \%$ conductive fillers.

The distribution of scattered points is not a real situation of electrically conductive composites; however, this method of particles distribution has been widely adopted for comparison with known percolation threshold values ${ }^{28-31}$.

\subsubsection{Algorithm:}

- generation of the insulating matrix formed only by elements "0" (Figure 3a);

- random generation of a pair of coordinates $(\mathrm{i}, \mathrm{j})$ representing the position of conductive element "1" in the matrix. 
- analysis whether the random position is occupied or not by a conductive element. If not, the position turns the number "0" into "1" (insulating - conductor); if yes, the generator processes a new number probably in a different position;

- maintenance of generation of conductive elements until the number of elements, which represents the desired volume fraction of conductive fillers, is found (Figure 3b).

After the distribution of conductive elements, it was necessary to analyze the generated matrix and verify if the percolation value was enough to form a continuous path of fillers that allows the movement of electrons and becomes composite conductor. Thus, the simulator analyzed the interconnectivity of the conductive elements. This procedure was performed with the following algorithm:

- scanning the first line to conductive elements identification ("1"). When found, the program transforms it on value "2" (Figure 3c);

- analysis of the existence of a neighbor around this point that is also a conductor element ("1"). After that the program also transforms these neighboring connected elements to value " 2 ". If it does not occur, the simulator performs the same procedure for another conductor element (Figure 3d).

- after complete scanning and processing of the matrix for connected elements, the occurrence in the last line of elements " 2 " is verified. If this happens, the frequency of appearance is equal to the number of pathways percolated in the system (Figure 3e).

The structure configuration defined by the simulator reflects the conditions for the percolation as described by the theory and the $p_{\mathrm{c}}$ values can be defined to points scattered randomly in the two-dimensional square lattice case. We found $p_{\mathrm{c}}=59.3 \%$. The aim at this stage is mainly to vary the matrix size, $n$, to verify the simulator randomness capacity. As the simulator is representative of fillers with high aspect ratio (ar), the matrix will have to present high $n$ values, so that not only ar, but also the concentration of values less than $1 \%$ can be represented by scattered points. As a result of this evaluation of the simulator capacity, the $p_{c}$ values for two-dimensional square lattices were simulated for 100 consecutive matrices and the results obtained are reported in Table 2.

Analyzing the results in Table 2 it can be said that the simulator describes, with confidence above $95 \%$, that, for a matrix with edge $n$ equal to $10000, p_{\text {c }}$ has values varying between 59.25 and $59.5 \%$, that is, very close to $59.3 \%$. This $n$ value will also enable ar representation above 1000, possible for CNT, and will generate the possibility of allocating 100,000,000 sites, essential for the simulation of small concentrations. For these reasons, the value $n=10,000$ was defined for the remaining simulations carried out in this study.

Before discussing the results for systems with single and hybrid fillers, a fundamental fact in the interpretation of later results should be emphasized, the dimensionality of simulated network. It is known in the literature that percolation values are tabulated ${ }^{9}$, and for different types of network geometry, a significant decrease of this threshold occurs when comparing the change in dimension of the network formation, from $2 \mathrm{D}$ to $3 \mathrm{D}$. In the case of scattered points for square and cubic networks, the $p_{\mathrm{c}} 2 \mathrm{D}$ value of $59.3 \%$, found by the simulator, should be closer to $31.1 \%$ if it was developed for 3D system formation. This reduction should be considered when comparing the experimental results with simulated results.

\subsubsection{Simulation of composite with Carbon Nanotubes}

In this part of the study, the simulator was adjusted to calculate the percolation threshold of nanocomposites as discretized fibers. For the simulation, entering the information as concentration and aspect ratio of the conductive fillers was necessary. The way of nanotubes random distribution was performed by the Monte Carlo method in accordance with algorithm. The provision of nanotubes was considered to be random and oriented at $45^{\circ}$, based on the excluded volume model. Figure 4 shows the filler real morphology (Figure 4a) and the individual CNT simulated model (Figure 4b).

The fiber diameter was defined as unitary, so that the $a r$ value entered into the programming was the CNT length. However, as shown in Figure 4, the zig-zag configuration, that is, the discretization of nanotubes represented a diameter with two points, which would double the tubes length. To minimize this length effect and to ensure nanotubes concentration in the matrix, Equation 3, based on the excluded volume model, was applied to determine the CNT number entered for percolation $\left(N_{\mathrm{c}, \mathrm{CNT}}\right)$.

$$
N_{c, C N T}=n^{2} \cdot V_{C N T} /(a r / 2)
$$

$n=$ size in points of matrix edge

$V_{\mathrm{CNT}}=$ volumetric fraction of CNT simulated $a r=($ aspect ratio) .

Table 2. Number of percolation in 100 simulations for different size matrices.

\begin{tabular}{lcccc}
\hline Number of Percolation in 100 Simulations & \multicolumn{5}{c}{ Filler concentration in the Matrix (\%) } \\
\hline Matrix Size $(n)$ & $\mathbf{5 9}$ & $\mathbf{5 9 . 2 5}$ & $\mathbf{5 9 . 5}$ & $\mathbf{6 0}$ \\
\hline 10 & 27 & 38 & 45 & 65 \\
100 & 34 & 36 & 52 & 80 \\
1000 & 30 & 55 & 68 & 96 \\
10000 & 44 & 96 & 100 & 100 \\
\hline
\end{tabular}




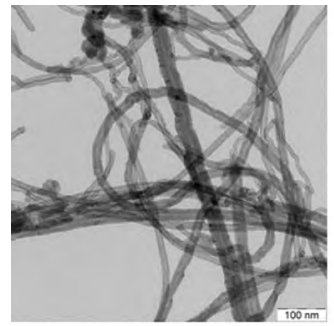

$\begin{array}{llllllllllllllll}0 & \cdots & 0 & 0 & 0 & 0 & 0 & 0 & 0 & 0 & 0 & 0 & 0 & 0\end{array}$ $\begin{array}{ccccccccccccccc}\ddots & \ddots & 0 & 0 & 0 & 0 & 0 & 0 & 0 & 0 & 0 & 0 & 0 & 0 \\ 0 & 0 & 0 & 1 & 0 & 0 & 0 & 0 & 0 & 0 & 0 & 0 & 0 & 0\end{array}$ $\begin{array}{llllllllllllllllllll}0 & 0 & 0 & 1 & 1 & 0 & 0 & 0 & 0 & 0 & 0 & 0 & 0 & 0\end{array}$ $\begin{array}{lllllllllllllllllllll}0 & 0 & 0 & 0 & 1 & 1 & 0 & 0 & 0 & 0 & 0 & 0 & 0 & 0\end{array}$ $\begin{array}{llllllllllllllllllll}0 & 0 & 0 & 0 & 0 & 1 & 1 & 0 & 0 & 0 & 0 & 0 & 0 & 0\end{array}$

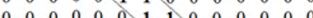
$\begin{array}{llllllllllllllllll}0 & 0 & 0 & 0 & 0 & 1 & 1 & 0 & 0 & 0 & 0 & 0 & 0\end{array}$

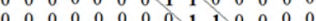

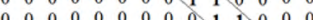
$\begin{array}{lllllllllllllllllllll}0 & 0 & 0 & 0 & 0 & 0 & 0 & 0 & 0 & 0 & 1 & 0 & 0 & 0 & 0\end{array}$ $\begin{array}{lllllllllllllllll}0 & 0 & 0 & 0 & 0 & 0 & 0 & 0 & 0 & 0 & 1 & 1 & 0 & 0\end{array}$ $\begin{array}{lllllllllllllllllll}0 & 0 & 0 & 0 & 0 & 0 & 0 & 0 & 0 & 0 & 0 & 1 & 1 & 0\end{array}$ $\begin{array}{llllllllllllllll}0 & 0 & 0 & 0 & 0 & 0 & 0 & 0 & 0 & 0 & 0 & 0 & 1 & 1\end{array}$ $\begin{array}{lllllllllllllll}0 & 0 & 0 & 0 & 0 & 0 & 0 & 0 & 0 & 0 & 0 & 0 & 0 & 1 \\ 0 & 0 & 0 & 0 & 0 & 0 & 0 & 0 & 0 & 0 & 0 & 0 & 0 & 0 & 0\end{array} 45^{\circ}$ 此

Figure 4. (a) Morphology and (b) Scheme of volume representation of CNT structure in simulator perspective.

The ar division by the constant correction 2 minimizes the diameter effect on the concentration, turns it into unitary, and does not influence the CNT contact capacity created by the simulator.

Moreover, it was necessary to determine which core model would be used in simulations, the "hard-core" model, which prevents two particles from occupying the same space or the "soft-core" model that allows this to occur. Relying on the fact that overlapping favorably influences the simulation time, the "soft-core" model was used overlapping of fillers in the two-dimensional space is allowed.

One factor that should also be considered is ar polydispersity of nanotubes and particles distribution ${ }^{32,33}$. Due to the difficulty in representing this polydispersity in the programming, the average size was taken as a basis for calculating ar of particles in most models described in the literature ${ }^{10}$. However, it is important to point out that in dispersion processes, such as sonification, the percolation threshold is influenced by the time, the mode and the power used in the dispersion of the fillers that will directly influence in the aspect ratio due the possible break of the nanotubes that can increase the polidispersivity. Therefore major errors can occur when average size was taken as a basis for calculating ar of particles.

As there is an influence of dispersion and the sizes of conductive filler in percolation threshold, to analyze this effect, simulations were performed for different ar values, varying filler volume concentration $\left(V_{\mathrm{CNT}}\right)$ in the matrix.
The percolation success (S) was evaluated in 100 successive simulations and a $p_{c}$ value was admitted when success ensured confidence above $95 \%$. Table 3 shows the results.

Table 3 shows that the simulator presented consistency in the simulation results, as by increasing $V_{\mathrm{CNT}}$ values, the success, $\mathrm{S}$, also increases, reaching values of $100 \%$ percolated matrices when the $p_{\mathrm{c}}$ value was simulated, highlighting $p_{\mathrm{c}}$ values, which showed a decrease with ar increasing. For better results viewing, an $a r$ versus $p_{\mathrm{c}}$ graph was constructed, which is shown in Figure 5. It can be seen that $p_{\mathrm{c}}$ is ar function.

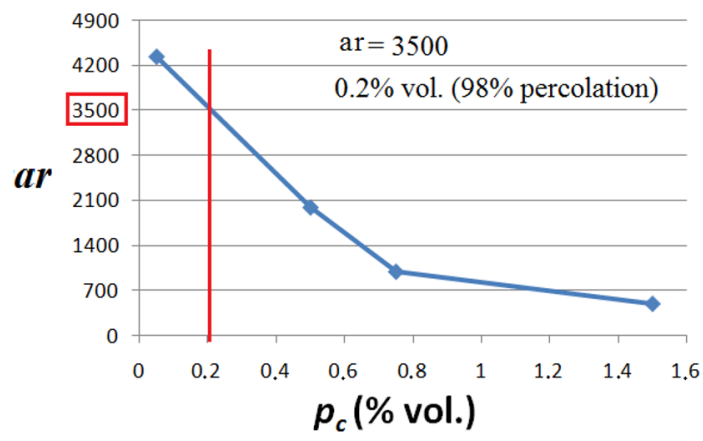

Figure 5. Graph of $a r$ values in $p$ function for 100 consecutive simulations.

Considering the configuration adopted for discretized nanotubes and randomly arranged at $45^{\circ}$ in the matrix, it was found that ar decrease in approximately 9 times, from 4348 to 500 , generating a $p_{c}$ increase of about 30 times, suggesting that for low critical concentration of percolation, a high ar was required by the simulator. Another observation is that for ar values below 500, the curve tends to present a plateau, indicating that small reductions in ar have a strong influence on $p_{\mathrm{c}}$ increase.

To check the program efficiency, the simulated results were compared with experimental values. The $p_{\mathrm{c}}$ value found experimentally for CNT/PMMA was $0.2 \%$ vol. For this value in the graph in Figure 5, the vertical line indicates that an ar 3500 would be necessary to achieve percolation. To verify this evidence, $V_{\mathrm{CNT}} 0.2 \%$ for ar 3500 was simulated and

Table 3. Simulation of pc,CNT with ar variable of CNT and evaluation of percolation success in 100 consecutive simulated matrices.

\begin{tabular}{|c|c|c|c|c|c|c|c|}
\hline \multicolumn{8}{|c|}{ Aspect Ratio (ar) } \\
\hline 4348* & & \multicolumn{2}{|c|}{2000} & \multicolumn{2}{|c|}{1000} & \multicolumn{2}{|c|}{500} \\
\hline$V_{\mathrm{CNT}}(\%$ vol. $)$ & $\mathbf{S}$ & $V_{\mathrm{CNT}}(\%$ vol. $)$ & $\mathbf{S}$ & $V_{\mathrm{CNT}}(\%$ vol. $)$ & $\mathbf{S}$ & $V_{\mathrm{CNT}}(\%$ vol. $)$ & $\mathbf{S}$ \\
\hline 0.01 & 0 & 0.0125 & 0 & 0.125 & 0 & 0.5 & 0 \\
\hline 0.0125 & 12 & 0.05 & 0 & 0.25 & 3 & 1 & 4 \\
\hline 0.025 & 60 & 0.1 & 12 & 0.5 & 51 & 1.25 & 45 \\
\hline 0.05 & 96 & 0.25 & 88 & 0.75 & 97 & 1.5 & 96 \\
\hline 0.125 & 100 & 0.5 & 98 & 1 & 100 & 1.75 & 98 \\
\hline 0.25 & 100 & 1 & 100 & 2.5 & 100 & 2.5 & 100 \\
\hline 0.5 & 100 & 2.5 & 100 & 5 & 100 & 5 & 100 \\
\hline
\end{tabular}

*Aspect ratio based on CNT provider data and study of COELHO et al., ${ }^{24}$ 
percolation was confirmed, S, on 98 of 100 matrices created (Figure 5). Therefore, it can be assumed that the program determined a $p_{c}$ value of $0.2 \%$, also found experimentally for $a r$ below the number provided by the CNT manufacturer. These results show that the simulator generated consistent values in $p_{\mathrm{c}}$ determination compared with those found in the literature ${ }^{10}$ and evaluated by theoretical approach of random particle distribution. Some studies state low $p_{c}$ values with low ar values ${ }^{11-13}$. This difference occurred because the simulation was performed in $2 \mathrm{D}$, resulting in values higher than would be the actual condition, 3D, where the possibility of contact between the particles would be higher, thus requiring lower fillers concentrations to the percolation. Celzart et al., ${ }^{11}$, following this approach, calculated the percolation threshold in $3 \mathrm{D}$ for compounds containing CNT randomly oriented in the epoxy matrix, to aspect ratio of 500 , and found a $0.5 \%$ critical mass concentration. This result is consistent with $p_{\mathrm{c}}$ experimental value of CNT/PMMA of $0.2 \%$ vol. ${ }^{17}$, which when converted to mass value is $0.45 \%$. This suggests the suitability of the current $3 \mathrm{D}$ simulator may be more representative when determining the percolation threshold.

\subsubsection{Simulation of composites with Carbon Black}

For CB systems, adjustments were made in the simulator based on the same principles of random distribution of particles; however, the program was used to calculate the percolation threshold of aggregated spherical particles. For the simulation, defining the geometry and the household size was necessary, in addition to conductive fillers concentration. The process for distribution of CB spherical particles (considered circular - 2D) was similar to the fibers process; however, the difficulty was in defining the geometry of aggregates of particles. The primary particle size and surface area, usually defined by the supplier, are not sufficient to describe the distribution phenomenon in polymer matrices. It is known that the provision of these particles is "grape-like", with different aggregate sizes ${ }^{34}$. This geometry precludes representative simulation of this primary structure, because, if the circular unitary particles of $\mathrm{CB}$ were randomly placed by the simulator, this trend of aggregation would be hardly expected. To solve this problem, the structural dimension of the aggregate was assumed to be multiple of unitary points (1 filled site is 10 $\mathrm{nm}$ ), related to the diameter on CNT simulation. Defining the geometry of the aggregate structure was fundamental when simulating $\mathrm{CB}$, as this would influence both the extent of contact between the fillers and the $\mathrm{CB}$ concentration in the matrix. This dispersion behavior of the spherical particles in forming the structure is shown in Figure 6, and allowed the definition of the aggregate structure and a model that could represent the exclusion of voids.

The literature usually defines CB geometry of aggregates as spherical ${ }^{10}$; however, in the simulator programming, square-shaped geometry was the one that best suited the possibility of representing the branched structure. Besides it still has the capability of contact when the square side was defined with the same size of the particle diameter. The square geometry was chosen because it represents the dimensional limit of the micrograph Figure 6a. Thus, one can evaluate how a real square plot of material would be summated by the program. Moreover, as there is no separation of individual particles and the structure is highly branched, there is a significant void area that interferes in the generation of an average geometry for the simulation. Therefore, these gaps should be disregarded for the simulator, since the squares generated will have the same contact ability of the branched structure, but are directly interfering in the matrix composite concentration. To consider this effect the excluded volume model was taken as base, and it was defined that, in two dimensions, the excluded area of the aggregate is the square area. However, this square area cannot be representative of
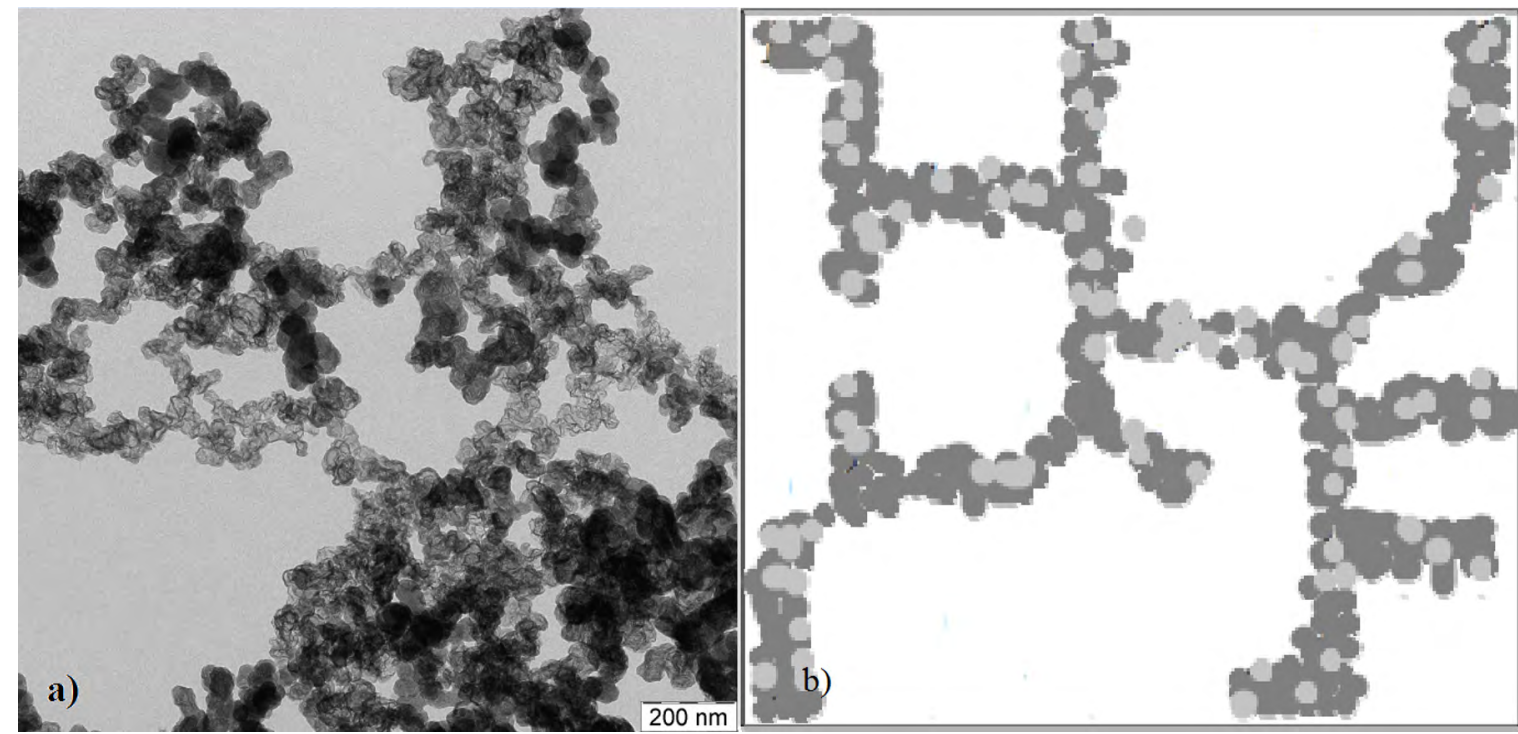

Figure 6. (a) Morphology of CB and (b) Representation of CB structure. 
CB concentration, and a correction factor was defined in Equation 4, designed for calculating the number of aggregates in the matrix, $N_{\mathrm{c}, \mathrm{CB}}$

$$
N_{c, C B}=n^{2} \cdot V_{C B} /\left(L_{q}^{2} \cdot \alpha^{-1}\right)
$$

$n=$ size in points of matrix edge

$V_{\mathrm{CB}}=$ simulated volume fraction

$L_{q}=$ size in square side points.

$\alpha=$ correction factor, which defines the ratio between the total concentration of the square (total number of points) and the concentration of spaces filled by aggregated spherical particles (number of points considered as concentration).

Figure 7 shows the aggregate scheme in the simulator perspective (Figure 7a) and representative $\alpha$ ratio of branched particle, responsible for correction of $\mathrm{CB}$ concentration in the matrix (Figure 7b).

Another difficulty in simulating the CB system was to determine the square size that was representative of the agglomerates and the representative $\alpha$ value of the fillers concentration. Therefore, the simulation was done to define percolation thresholds, $p_{\mathrm{c}}$, based on the variation of some $\alpha$ values for different square dimensions. Table 4 shows the results.

By adopting $\alpha=0$, that is, by considering a square filled by conductive fillers, the simulator basically behaved in the same proportion of scattered points found in the previous simulator test, that is, $p_{\mathrm{c}}$ values close to $60 \%$ vol. The $\alpha$ values equal to 5 and 10 represent, respectively, that the square is filled with $1 / 5$ and $1 / 10$ of conductive fillers, as the remainder of the square filling are empty spaces. Thus, the excluded area of CB cluster, which is closely connected to contact capacity between fillers, will be the same as the
Table 4. Percolation threshold values for $\mathrm{CB}$ systems: variation of $L_{q}$ and value of $\alpha$.

\begin{tabular}{lccc}
\hline$\alpha$ & 0 & 5 & 10 \\
\hline$L_{q}$ (points) & $p_{\mathrm{c}}(\%$ vol. $)$ & $p_{\mathrm{c}}(\%$ vol. $)$ & $p_{\mathrm{c}}(\%$ vol. $)$ \\
$\mathbf{5 0}$ & 60 & 45 & 35 \\
$\mathbf{1 0 0}$ & 55 & 30 & 25 \\
$\mathbf{2 0 0}$ & 50 & 20 & 12.5 \\
$\mathbf{4 0 0}$ & 60 & 17.5 & 7.5 \\
\hline
\end{tabular}

square. Higher values of $\alpha$ indicate higher values of $N_{\mathrm{c}}$, that is, the number of particles dispersed in the matrix increases, allowing a greater possibility of forming a pathway for electric current passage, generating a consequent reduction of percolation threshold. In order to evaluate the best $\alpha$ correction and to verify the program efficiency, the simulated results were compared to the percolation threshold found experimentally. The value of $\alpha=10$ applied to the model was the best concentration simulated correction, since the simulated $p^{\mathrm{c}}$ value of $7.5 \%$ vol. for $L_{q}$ of 400 was the one closest to the experimental value of $1.5 \%$ vol., even though it was 5 times lower. Therefore, it is assumed that this $\alpha$ value is a valid relation, and the graph of Figure 8, may suggest a better approximation of these results.

Emphasizing the direct association of $L_{q}$ with $p_{c}$, one may suggest that for the simulated value be even closer to the experimental, the aggregate size should have a diameter greater than 400 points, that is, an actual value of $4 \mu \mathrm{m}$ side, if again the enforced relationship for CNT is considered: 1 point is equivalent to $1 \mathrm{~nm}$. When comparing this value with respect to the primary particle diameter, $30 \mathrm{~nm}$, the aggregate side would have thousands of unitary spheres to complete the size in micrometers, something that at first seemed impossible.

$\begin{array}{llllllllllllllll}0 & \cdots & 0 & 0 & 0 & 0 & 0 & 0 & 0 & 0 & 0 & 0 & 0 & 0 & 0 & 0 \\ \vdots & \ddots & 0 & 0 & 0 & 0 & 0 & 0 & 0 & 0 & 0 & 0 & 0 & 0 & 0 & 0 \\ 0 & 0 & 0 & 0 & 0 & 0 & 0 & 0 & 0 & 0 & 0 & 0 & 0 & 0 & 0 & 0 \\ 0 & 0 & 0 & 0 & 0 & 0 & 0 & 0 & 0 & 0 & 0 & 0 & 0 & 0 & 0 & 0 \\ 0 & 0 & 0 & 0 & 0 & \mathbf{1} & \mathbf{1} & \mathbf{1} & \mathbf{1} & \mathbf{1} & \mathbf{1} & \mathbf{1} & \mathbf{1} & \mathbf{1} & \mathbf{1} & 0 \\ 0 & 0 & 0 & 0 & 0 & \mathbf{1} & \mathbf{1} & \mathbf{1} & \mathbf{1} & \mathbf{1} & \mathbf{1} & \mathbf{1} & \mathbf{1} & \mathbf{1} & \mathbf{1} & 0 \\ 0 & 0 & 0 & 0 & 0 & \mathbf{1} & \mathbf{1} & \mathbf{1} & \mathbf{1} & \mathbf{1} & \mathbf{1} & \mathbf{1} & \mathbf{1} & \mathbf{1} & \mathbf{1} & 0 \\ 0 & 0 & 0 & 0 & 0 & \mathbf{1} & \mathbf{1} & \mathbf{1} & \mathbf{1} & \mathbf{1} & \mathbf{1} & \mathbf{1} & \mathbf{1} & \mathbf{1} & \mathbf{1} & 0 \\ 0 & 0 & 0 & 0 & 0 & \mathbf{1} & \mathbf{1} & \mathbf{1} & \mathbf{1} & \mathbf{1} & \mathbf{1} & \mathbf{1} & \mathbf{1} & \mathbf{1} & \mathbf{1} & 0 \\ 0 & 0 & 0 & 0 & 0 & \mathbf{1} & \mathbf{1} & \mathbf{1} & \mathbf{1} & \mathbf{1} & \mathbf{1} & \mathbf{1} & \mathbf{1} & \mathbf{1} & \mathbf{1} & 0 \\ 0 & 0 & 0 & 0 & 0 & \mathbf{1} & \mathbf{1} & \mathbf{1} & \mathbf{1} & \mathbf{1} & \mathbf{1} & \mathbf{1} & \mathbf{1} & \mathbf{1} & \mathbf{1} & 0 \\ 0 & 0 & 0 & 0 & 0 & \mathbf{1} & \mathbf{1} & \mathbf{1} & \mathbf{1} & \mathbf{1} & \mathbf{1} & \mathbf{1} & \mathbf{1} & \mathbf{1} & \mathbf{1} & 0 \\ 0 & 0 & 0 & 0 & 0 & \mathbf{1} & \mathbf{1} & \mathbf{1} & \mathbf{1} & \mathbf{1} & \mathbf{1} & \mathbf{1} & \mathbf{1} & \mathbf{1} & \mathbf{1} & 0 \\ 0 & 0 & 0 & 0 & 0 & \mathbf{1} & \mathbf{1} & \mathbf{1} & \mathbf{1} & \mathbf{1} & \mathbf{1} & \mathbf{1} & \mathbf{1} & \mathbf{1} & \mathbf{1} & 0 \\ 0 & 0 & 0 & 0 & 0 & 0 & 0 & 0 & 0 & 0 & 0 & 0 & 0 & 0 & 0 & 0 \\ 0 & 0 & 0 & 0 & 0 & 0 & 0 & 0 & 0 & 0 & 0 & 0 & 0 & 0 & 0 & 0\end{array}$

$$
L_{q}
$$

$\begin{array}{llllllllllllllll}0 & \cdots & 0 & 0 & 0 & 0 & 0 & 0 & 0 & 0 & 0 & 0 & 0 & 0 & 0 & 0 \\ \vdots & \ddots & 0 & 0 & 0 & 0 & 0 & 0 & 0 & 0 & 0 & 0 & 0 & 0 & 0 & 0 \\ 0 & 0 & 0 & 0 & 0 & 0 & 0 & 0 & 0 & 0 & 0 & 0 & 0 & 0 & 0 & 0 \\ 0 & 0 & 0 & 0 & 0 & 0 & 0 & 0 & 0 & 0 & 0 & 0 & 0 & 0 & 0 & 0 \\ 0 & 0 & 0 & 0 & 0 & \mathbf{1} & \mathbf{1} & 0 & 0 & \mathbf{1} & 0 & 0 & 0 & 0 & \mathbf{1} & 0 \\ 0 & 0 & 0 & 0 & 0 & 0 & \mathbf{1} & 0 & 0 & \mathbf{1} & 0 & 0 & 0 & 0 & \mathbf{1} & 0 \\ 0 & 0 & 0 & 0 & 0 & 0 & \mathbf{1} & \mathbf{1} & \mathbf{1} & \mathbf{1} & 0 & 0 & 0 & 0 & \mathbf{1} & 0 \\ 0 & 0 & 0 & 0 & 0 & 0 & \mathbf{1} & 0 & 0 & \mathbf{1} & 0 & 0 & 0 & \mathbf{1} & 0 & 0 \\ 0 & 0 & 0 & 0 & 0 & 0 & 0 & 0 & 0 & \mathbf{1} & \mathbf{1} & \mathbf{1} & \mathbf{1} & 0 & 0 & 0 \\ 0 & 0 & 0 & 0 & 0 & 0 & \mathbf{1} & 0 & 0 & \mathbf{1} & 0 & 0 & \mathbf{1} & \mathbf{1} & \mathbf{1} & 0 \\ 0 & 0 & 0 & 0 & 0 & 0 & \mathbf{1} & \mathbf{1} & \mathbf{1} & 0 & \mathbf{1} & 0 & \mathbf{1} & 0 & 0 & 0 \\ 0 & 0 & 0 & 0 & 0 & \mathbf{1} & 0 & 0 & 0 & 0 & 0 & 0 & \mathbf{1} & \mathbf{1} & \mathbf{1} & 0 \\ 0 & 0 & 0 & 0 & 0 & \mathbf{1} & 0 & 0 & 0 & 0 & 0 & 0 & \mathbf{1} & 0 & 0 & 0 \\ 0 & 0 & 0 & 0 & 0 & \mathbf{1} & 0 & 0 & 0 & 0 & 0 & \mathbf{1} & \mathbf{1} & 0 & 0 & 0 \\ 0 & 0 & 0 & 0 & 0 & 0 & 0 & 0 & 0 & 0 & 0 & 0 & 0 & 0 & 0 & 0 \\ 0 & 0 & 0 & 0 & 0 & 0 & 0 & 0 & 0 & 0 & 0 & 0 & 0 & 0 & 0 & 0\end{array}$

b)

Figure 7. Scheme of volumetric representation of CB aggregate in the matrix: (a) aggregate in the simulator perspective; (b) aggregate after applying $\alpha$ correction factor. 


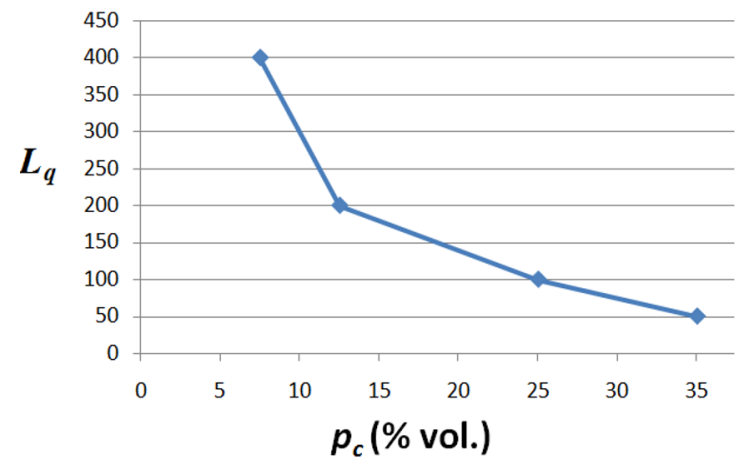

Figure 8. $L_{q}$ in function of $p_{\mathrm{c}}$ for 100 consecutive simulations for the correction factor $\alpha=10$.

However, reviewing the work of Hauptmanc et al. ${ }^{35}$ for $\mathrm{CB}$ Printex XE 2 B, it was found that the average aggregate size was approximately $8 \mu \mathrm{m}$ in diameter, two times greater than the 400 simulated points. This would probably lead to a smaller $p_{\mathrm{c}}$ value. To evaluate this assumption, the linearization of a curve of $L_{q}$ as a function of $p_{c}$, Fig 8, was performed. This applied mathematical analysis on a logarithmic scale allowed adjusting a $p_{\mathrm{c}} 5 \%$ vol. value to a $8.6 \mu \mathrm{m}$ diameter, near to the found in the literature ${ }^{35}$, for a linear regression coefficient, $R$, close to $99 \%$ as shown in Figure 9.

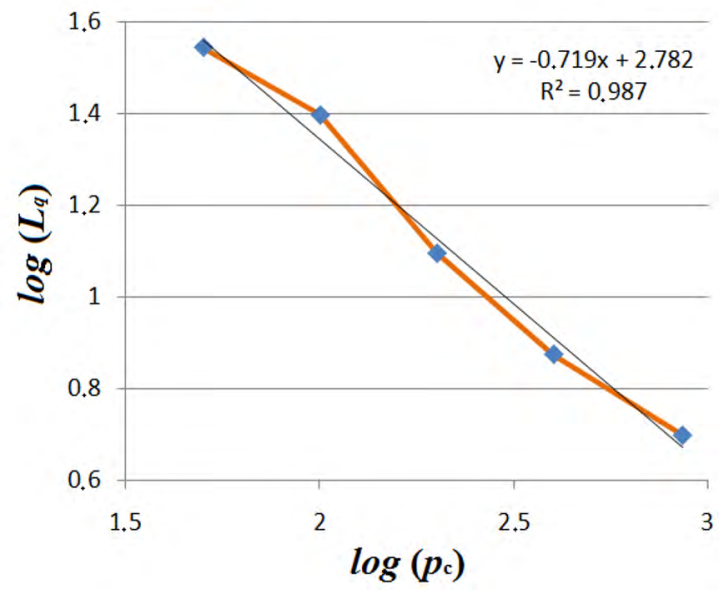

Figure 9. Linear adjustment of $\log$ curve $\left(L_{q}\right) v s \log \left(p_{\mathrm{c}}\right)$ for CB.

Thus, in the simulation the $8.6 \mu$ m diameter was adopted, which converted into points resulted in $L_{q}$ of 860 occupied sites. The $5 \%$ vol. was simulated and success was obtained in 97 of 100 simulated matrices. This simulated value was the closest to the experimental value, and therefore adopted for calculating the percolation threshold of hybrid nanocomposites.

\subsubsection{Simulation of Hybrid Nanocomposites}

At this stage of the work, the percolation threshold determination was developed for hybrid fillers, $p_{c^{\prime} \mathrm{H}}$, by a simulator in FORTRAN, by applying the Monte Carlo method. Therefore, defining the simulator capacity to assess the random dispersion of individual fillers was necessary. Previously items confirmed that the program has shown efficiency in the approximation of values of $p_{c^{\prime} \mathrm{CNT}}$ and $p_{\mathrm{c}^{\prime} \mathrm{CB}}$ experimental of individual fillers, since the results were close to experimental values in the literature, after ar and $L_{q}$ definition, for CNT, and CB. Based on this analysis, to study $p_{c^{\prime} \mathrm{H}}$, the process was defined based on values of $p_{\mathrm{c}^{\prime} \mathrm{CNT}}$ and $p_{\mathrm{c}^{\prime} \mathrm{CB}}$ simulated and assumed to be $0.2 \%$ vol. with aspect ratio of 3500 for $\mathrm{CNT}$, and $5 \%$ vol. for the aggregate size of 860 sites for CB. Experimental comparison for evaluating the simulator capacity to meet the percolation threshold for the hybrid system was subsequently performed. In this stage the values of simulated concentrations were the same calculated by the Sun et al. ${ }^{5}$, model, described in Equation 1 , and shown in Table 1.

To evaluate the simulator efficiency, the simulated results were compared with the experimental values, Figure 3, and are shown in Table 5. Taking these settings as bases, samples from 1 to 4 , which satisfy the condition $V_{\mathrm{CNT}} / p_{\mathrm{c}, \mathrm{CNT}}+V_{\mathrm{CB}} /$ ${ }_{\mathrm{pc}, \mathrm{CB}}=1$ of percolation proposed in the Sun model, and for samples from 5 to 8 , which are below the provided in the model, $V_{\mathrm{CNT}} / p_{\mathrm{c}, \mathrm{CNT}}+V_{\mathrm{CB}} / p_{\mathrm{c}, \mathrm{CB}}<1$.

One has to point out that the values calculated for samples 1-4, by the Sun's model, were obtained from the experimental percolation threshold, $p_{\mathrm{c}, \mathrm{CNT}}$ e $p_{\mathrm{c}, \mathrm{CB}}, 0.2 \%$ vol. and $1.5 \%$ vol. respectively. The simulated $p_{c, C B}$ value for the CB was found to be $5.0 \%$ vol., which was higher than the experimental $p_{\mathrm{c}, \mathrm{CB}}$. As the same algorithm was applied in the hybrids simulation, it would be expected that no sample of hybrid system would success in percolation, because the equation 1 (Sun's model) would not be fulfilled. However, when analyzing the results of Table 5 , with $95 \%$ confidence it was observed that samples 3 and 4 percolated, even though with values well below the experimental percolation threshold, showing that the program provides the synergism effect between fillers. In order to perceive the synergetic results of the hybrid systems Table 5 and Figure 3 should be analyzed simultaneously. When comparing, for example, sample 3, where the simulated percolation was $0.11 \%$ vol $\mathrm{CNT}+0.64 \%$ vol. $\mathrm{CB}=0.75 \%$ vol, with simulated values for individual fillers, $0.2 \%$ vol. CNT and $5 \%$ vol. $\mathrm{CB}$, one can observe a reduction of almost $1 / 2$ the $\mathrm{CNT}$ concentration and $1 / 8$ the $\mathrm{CB}$ concentration, still describing the hybrid nanocomposite as a conductor.

Another important aspect was to reflect on the importance of high CNT aspect ratio on simulated percolation, since as well as in the experimental part, the CNT has served as a coupling agent, as when it was in concentration an order of magnitude lower than its individual percolation, the simulation did not determine success with the adopted $95 \%$ confidence. Therefore, it is reasonable to assume that the structure proposed by the simulator by the Monte Carlo 
Table 5. Percolation Success (S) of 100 simulations for hybrid nanocomposites.

\begin{tabular}{lcccc}
\hline Samples & $V_{\mathrm{CNT}}(\%$ vol. $)$ & $V_{\mathrm{CB}}(\% \mathrm{vol})$. & Total (\%vol.) & $\mathrm{S}$ \\
\hline $\mathbf{1}$ & 0.03 & 1.27 & 1.3 & 27 \\
$\mathbf{2}$ & 0.06 & 1.07 & 1.13 & 79 \\
$\mathbf{3}$ & 0.11 & 0.64 & 0.75 & 95 \\
$\mathbf{4}$ & 0.17 & 0.21 & 0.38 & 96 \\
$\mathbf{5}$ & 0.01 & 1.21 & 1.22 & 0 \\
$\mathbf{6}$ & 0.03 & 0.91 & 0.94 & 29 \\
$\mathbf{7}$ & 0.06 & 0.6 & 0.66 & 35 \\
$\mathbf{8}$ & 0.11 & 0.3 & 0.41 & 47 \\
\hline
\end{tabular}
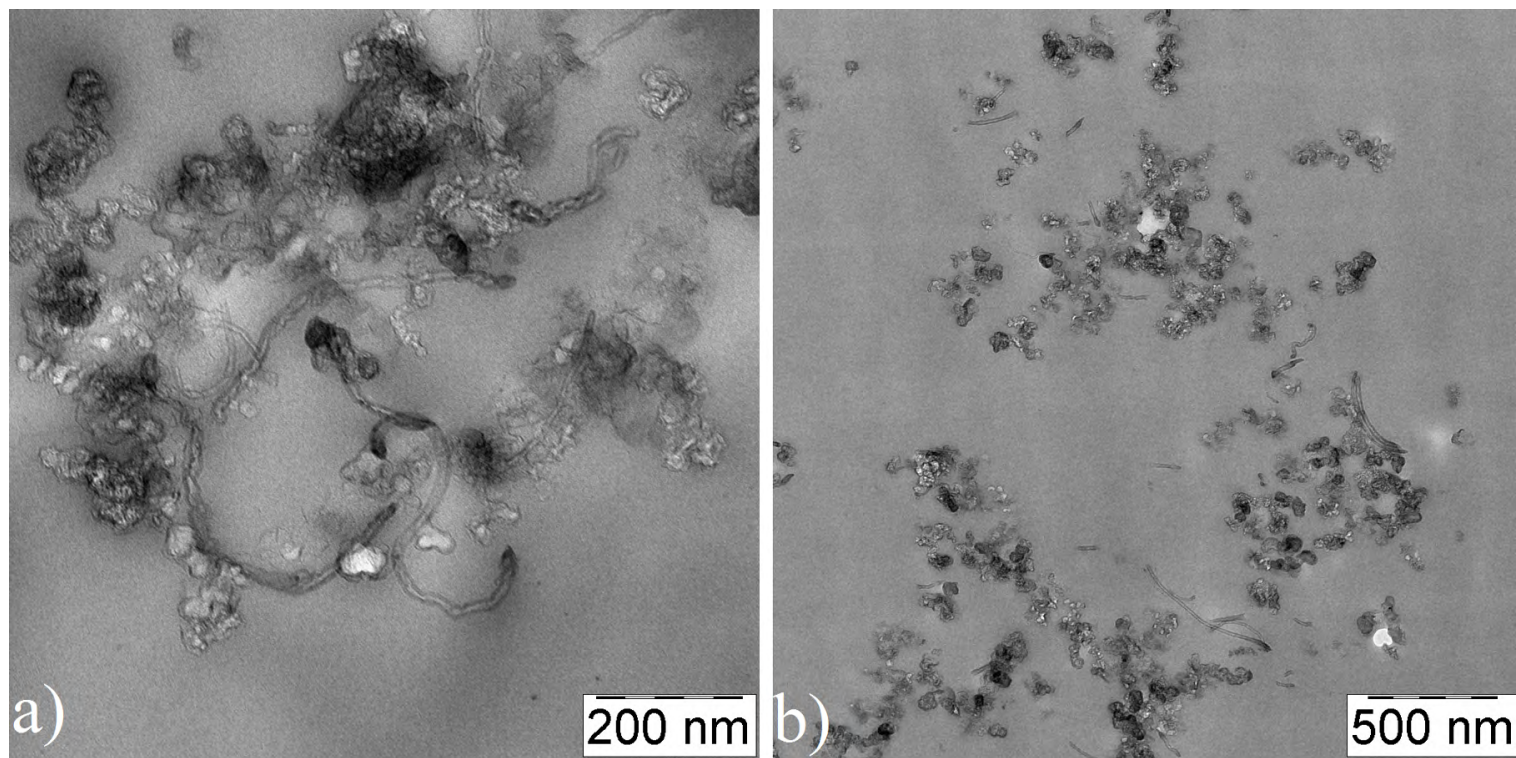

Figure 10. TEM micrographs for MCFFS (a) conductive networks; (b) larger view of scattered CB and CNT.

method randomness approximates experimental $p_{\mathrm{c}, \mathrm{H}}$ value, indicating a good prediction of the threshold for hybrid fillers. The morphology of a specific the sample containing 0.06 vol. (\%) of CNT and 1.07 vol. (\%) of CB was analyzed by TEM and the micrograph is presented in Figure 10. It represents the fillers interactions in the hybrid percolative morphology where the bridge effect of $\mathrm{CNT}$ to $\mathrm{CB}$ aggregates could be possible. $\mathrm{CB}$ aggregates and $\mathrm{CNT}$ is homogeneously scattered in the polymer matrix. This feature reveals CNT linking the CB aggregates forming conductive networks. These networks were also observed in other studies ${ }^{27,34,36}$. $\mathrm{CB}$ morphology is "grape-like", consisting of individual nanoparticles ${ }^{34}$. CNT, due its high aspect ratio, can act as conducting links between the CB particles ${ }^{34,37}$. In our study, we can describe the morphology as a "grape-cluster-like" 34 conductive network in which $\mathrm{CB}$ particles serve as grapes and $\mathrm{CNT}$ acts as branches.

\section{Final Remarks}

Conductive behavior of nanocomposites of carbon black, carbon nanotubes and their blends were studied. A program based on Monte Carlo method, supported on the excluded volume model was developed for two-dimensional systems. It was concluded to be promising to perform simulations for percolation threshold of hybrid systems. Simulated percolation threshold values for individual fillers were consistent with the experimental results and the literature. For hybrid systems, the simulator could predict the synergism between the fillers, which may suggest that an effective dispersion and good control of aspect ratio of the fillers can reproduce this effect in real conditions. However, it has also been discussed that considering average values of particles size can result in inaccuracies in the results. A future approach is a study including particle size polydispersity in programming. In addition, an expansion for 3D has the potential to better fit the simulator with the experimental reality. This can be a great contribution to the reduction of samples amount to be prepared in an experimental study.

\section{Acknowledgements}

The research was conducted with support from LNNano - LNLS- Brazilian Synchrotron Light Laboratory, CNPEM / 
ABTLuS / MCTI. The authors also thank to CNPq for financial support to the project and Semiconducting Components Center (CCS-Unicamp).

\section{References}

1. Bauhofer W, Kovacs JZ. A review and analysis of electrical percolation in carbon nanotube polymer composites. Composites Science and Technology. 2009;69(10):1486-1498

2. Clingerman LM, Weber EH, King JA, Schulz KH. Synergistic effect of fillers in electrically conductive nylon 6,6 and polycarbonate based resins. Polymer Composites. 2002;23(5):911-924. DOI: 10/1002/pc. 10488

3. Thongruang W, Spontak RJ, Balik CM. Correlated electrical conductivity and mechanical property analysis of high-density polyethylene filled with graphite and carbon fiber. Polymer. 2002;43(8):2279-2286.

4. Lux F. Models proposed to explain the electrical conductivity of mixtures made of conductive and insulating materials. Journal of Materials Science. 1993;28(2):285-301.

5. Sun Y, Luo S, Watkins K, Wong CP. Electrical approach to monitor the thermal oxidation aging of carbon black filled ethylene propylene rubber. Polymer Degradation and Stability. 2004;86(2):209-215. DOI: 10.1016/polymdegradstab.2004.04.2013

6. Socher R, Krause B, Hermasch S, Wursche R, Pötschke P. Electrical and thermal properties of polyamide 12 composites with hybrid fillers systems of multiwalled carbon nanotubes and carbon black. Composites Science and Technology. 2011;71(8):1053-1059.

7. Metropolis N. The beginning of the Monte Carlo Method. Los Alamos Science. 1987;Spec Iss:125-130.

8. Strümpler R, Glatz-Reichenbach J. Conducting polymers composites. Journal of Electroceramics. 1999;3(4):329-346.

9. Zallen RA. The Percolation Model. In: Zallen RA. The Physics of Amorphous Solids. Chap. 4. Hoboken: Wiley; 1983.

10. Balberg I, Anderson CH, Alexander S, Wagner N. Excluded volume and its relation to the onset of percolation. Physical Review B. 1984;30(7):3933-3943.

11. Celzard A, McRae E, Deleuze C, Dufort M, Furdin G, Marêché JF. Critical concentration in percolating systems containing a high-aspect-ratio filler. Physical Review B. 1996;53(10):62096214. DOI: 10/1103/PhyRevB.53.6209

12. Wu SH, Massaharuj I, Natsuki T, Ni QQ. Electrical Conduction and Percolation Behavior of Carbon Nanotubes/UPR Nanocomposites. Journal of Reinforced Plastics and Composites. 2006;25(18):1957-1966.

13. Bug AL, Safran SA, Webman II. Continuum Percolation of Rods. Physical Review Letters. 1985;54(13):1412-1415. DOI: 10.1103/PhysRevLett.54.1412

14. Berhan L, Sastry AM. Modeling percolation in high-aspectratio fiber systems. I. Soft-core versus hard-core models. Physical Review E. 2007;75(4 Pt 1):0411201. DOI: 10.1103/ PhyRevLett.75.041120
15. Berhan L, Sastry AM. Modeling percolation in high-aspect-ratio fiber systems. II. The effect of waviness on the percolation onset. Physical Review E. 2007;75(4 Pt 1):0411211. DOI: 10.1103/ PhysRevE.75.041121

16. Dalmas F, Dendievel R, Chazeau L, Cavaillé JY, Gauthier C. Carbon nanotube-filled polymer composites. Numerical simulation of electrical conductivity in three-dimensional entangled fibrous networks. Acta Materialia. 2006;54(11):2923-2931.

17. Coelho PHSL, Marchesin MS, Morales AR, Bartoli JR. Electrical percolation, morphological and dispersion properties of MWCNT/ PMMA nanocomposites, Materials Research. 2014;17(Supp1 1):127-132. DOI: 10.1590/S1516-14392014005000059

18. Coelho PL, Morales AR. Comparative Study of the Electrical Percolation and Dispersion of CB/PMMA Composites. In: XIV Latin American Symposium on Polymer; 2014 Oct 12-16; Porto de Galinhas, PE, Brazil.

19. Balberg I, Binenbaum N, Wagner N. Percolation Thresholds in the Three-Dimensional Sticks System. Physical Review Letters. 1984;52(17):1465-1468.

20. Al-Saleh M, Sundararaj U. Electromagnetic Interference (EMI) Shielding Effectiveness of PP/PS Polymer Blends Containing High Structure Carbon Black. Macromolecules Materials and Engineering. 2008;293:621-630.

21. Kirkpatrick S. Percolation and Conduction. Reviews of Modern Physics. 1973;45(4):574.

22. Sichel EK, Gittelman JI, Sheng P. Electrical properties of carbon-polymer composites. Journal of Electronics Materials. 1982;11(4):699-747.

23. Kim HM, Kim K, Lee SJ, Joo J, Yoon HS, Cho SJ, et al. Charge transport properties of composites of multiwalled carbon nanotube with metal catalyst and polymer: application to electromagnetic interference shielding. Current Applied Physics. 2004;4(6):577-580.

24. Schaefer D, Hurd A. In: Proceedings of the Symposium on the Chemistry and Physics of Composite Media; 1985; Toronto, ON, Canada. Pennington: The Electrochemical Society. p. $85-88,54-62$.

25. Keefer KD, Schaefer DW. Growth of Fractally Rough Colloids. Physical Review Letters. 1986;56(22):2376-2379.

26. García-Gutiérrez MC, Nogales A, Hernández JJ, Rueda DR, Ezquerra TA. X-ray scattering applied to the analysis of carbon nanotubes, polymers and nanocomposites. Óptica Pura y Aplicada. 2007;40(2):195-205.

27. Villmow T, Pegel S, Pötschke P, Wagenknecht U. Influence of injection molding parameters on the electrical resistivity of polycarbonate filled with multi-walled carbon nanotubes. Composites Science and Technology. 2008;68(3-4):777-789.

28. Newman MEJ, Ziff RM. Efficient Monte Carlo algorithm and high-precision results for percolation. Physical Review Letters. 2000;85(19):4104-4107. DOI: 10.1103/PhysRevLett.85.4104

29. Oliveira PMC, Nóbrega RA, Stauffer D. Corrections to finite size scaling in percolation. Brazilian Journal of Physics. 2003;33(3):616-618. DOI: 10.1590/S0103-97332003000300025 
30. Lee MJ. Complementary algorithms for graphs and percolation. Physical Review E. 2007;76(2 Pt 2):027702. DOI: 10.1103/ PhysRevE.76.027702

31. Lee MJ. Pseudo-random-number generators and the square site percolation threshold. Physical Review E. 2008;78(3 Pt 1):031131. DOI: 10.1103/PhysRevE.78.031131

32. Chatterjee AP. Percolation thresholds for rod-like particles: polydispersity effects. Journal of Physics: Condensed Matter. 2008;20(25):255250. DOI: 10.1088/0953-8984/20/25/255250

33. Mecke KR, Seyfried A. Strong dependence of percolation thresholds on polydispersity. Europhysics Letters. 2002;58(1):28.

34. Wen M, Sun X, Su L, Shen J, Li J, Guo S. The electrical conductivity of carbon nanotube/carbon black/polypropylene composites prepared through multistage stretching extrusion. Polymer. 2012;53(7):1602-1610.

35. Hauptman N, Vesel A, Ivanovski V, Gunde MK. Electrical conductivity of carbon black pigments.Dyes and Pigments. 2012;95(1):1-7

36. Lellinger D, Xu D, Ohneiser A, Skipa T, Alig I. Influence of the injection moulding conditions on the in-line measured electrical conductivity of polymer-carbon nanotube composites. Physics Status Solid B. 2008;245(10):2268-2271.

37. Lee JH, Jang YK, Hong CE, Kim NH, Li P, Lee HK. Effect of carbon fillers on properties of polymer composite bipolar plates of fuel cells. Journal of Power Sources. 2009;193(2):523-529. 